\title{
Producción científica nacional e internacional en drogas de diseño (1988-1997)
}

\author{
Aleixandre Benavent, R.*; Valderrama Zurián, J.C. **; Cervera, G.*** \\ *Instituto de Estudios Históricos y Documentales López Piñero (Universitat de València-CSIC). \\ **Unidad de Conductas Adictivas. Áreas 16 y 18 de Alicante. Conselleria de Sanitat i Consum. Generalitat Valenciana. Alicante. \\ ***Unidad de Desintoxicación. Hospital Clínico Universitario. Valencia.
}

Enviar correspondencia a :

Rafael Aleixandre Benavent. Instituto de Estudios Históricos y Documentales López Piñero (Universitat de València-CSIC).

Facultad de Medicina de Valencia. Avda. Blasco Ibañez, 17 46010-Valencia

\section{Resumen}

Introducción: El conocimiento de las fuentes de información científica es imprescindible para el abordaje del problema del consumo de drogas de diseño. El objetivo de este trabajo es analizar la producción científica nacional e internacional sobre esta conducta, a partir del análisis bibliométrico de las publicaciones.

Material y método: La producción científica sobre drogas de diseño se ha obtenido de las bases de datos IME, MEDLINE, ISOC y teseo durante el período 1988-1997. También se revisaron las referencias bibliográficas de los artículos recuperados en la base de datos IME. Los artículos obtenidos se distribuyeron por años, tipo documental, revista de publicación y temática, instituciones y países de procedencia, idiomas y temas tratados.

Resultados: En las revistas españolas se publicaron 34 artículos y en las extranjeras 2.181. Los principales aspectos tratados se refieren a la toxicidad, concepto y clasificación y actividad farmacológica de las drogas de diseño. Las áreas temáticas más productivas son la neuropsiquiatría, la farmacología y la medicina general e interna.

Conclusiones: El análisis muestra que existen escasos estudios experimentales y epidemiológicos publicados en España. Las revistas son de drogodependencias, temática general, neuropsiquiatría y farmacología, lo que pone de relieve la multidisciplinariedad de esta conducta adictiva. Predominan los estudios sobre la 1-metil-4-fenil-1,2,3,6-tetrahidropiridina y los análogos y derivados de la MDMA.

Palabras clave: drogas de diseño, fuentes de información, bibliometría, productividad científica, análisis de referencias

\section{Abstract}

Introduction: The knowledge of the scientific information sources is essential to approach the problem of the design drugs consumption. The objective of this work is directed to analyse the national and international scientific production about such behaviour, starting from the analysis bibliometric of the publications.

Material and method: The scientific production on design drugs is being obtained from the data base IME, MEDLINE, ISOC and teseo during the 1988/1997 period. It has been done checking the bibliographic references of the articles recovered from the data base IME. The articles obtained have been separated by year, documentation type, journals thematic, institutions, country of origin, language and subject-matter.

Results: In the spanish journals there has been published 34 articles and 2.181 in foreing journals. The main aspects treated make reference to toxicity, concept, classification and pharmacological activity of the design drugs. The thematic areas more productive are neuropsychiatry, pharmacological and general and internal medicine.

Conclusions: The analysis points out that its does exist minimal experimental and epidemiological studies published in Spain. The journals more productives are specialised in drug addiction, general thematic, neuropsychiatry and pharmacological, what underlines the multidisciplinary of this addictive behaviour. The studies of the 1-methyl-4-phenyl-1,2,3,6-tetrahydropyridine and of analogues and derived of MDMA predominate.

Key words: Design drugs, information sources, bibliometric, scientific productivity, analysis of references.

\section{INTRODUCCIÓN}

$\mathbf{L}$ os profesionales que atienden a pacientes usuarios de drogas de diseño suelen carecer de información científica sobre las características y efectos de estas drogas (mecanismos de acción, efectos farmacológicos, interacciones, efectos adversos, toxi- cidad, etc.). Otras drogas también ilegales, como opiáceos, alucinógenos, derivados del cannabis, cocaína y otros simpaticomiméticos, antes de su ilegalización han sido empleados con finalidad terapéutica, existiendo por ello un amplio conocimiento que permite conocer sus riesgos, así como tratar las complicaciones médicas o psiquiátricas derivadas de su consumo'. 
Resulta oportuno definir, en primer lugar, el concepto de droga de diseño, ya que el término se presta a muchas interpretaciones. La definición podría abarcar a todas aquellas sustancias sintéticas diseñadas en laboratorio en un intento de conseguir determinadas propiedades farmacológicas para potenciar su capacidad de ejercer efectos especiales ${ }^{2}$. En este sentido, se ha modificado la estructura química de muchos compuestos con el fin de mejorar, entre otras propiedades, su absorción, potencia o duración de su acción. Sin embargo, en la actualidad, el término suele restringirse a los derivados metoxilados sintéticos de la anfetamina que, en un principio, fueron diseñados por su posible potencial terapéutico, aunque en realidad carecen de esta utilidad en la especie humana y sólo presentan efectos alucinógenos, comportándose como estimulantes de tipo anfetamínico y con efectos psicoticomiméticos similares a los de la dietilamida del ácido lisérgico (LSD) o a la mescalina (alcaloide natural del cactus peyote $)^{3}$.

Los derivados anfetamínicos alucinógenos (d-anfetamina, metanfetamina, éxtasis, píldora del amor, eva, 4-metil-2,5-dimetoxianfetamina o DOM, píldora de la paz) constituyen el grupo de sustancias químicas que con más propiedad pueden denominarse "drogas de diseño". Todos ellos producen efectos similares, aun- que con matices subjetivos en relación con el sujeto que las ingiere y la vía de administración (oral, intravenosa o intranasal) ${ }^{2}$.

El término fue citado por primera vez para referirse a una serie de sustancias químicas análogas a las drogas ilícitas, sintetizadas con finalidad recreativa y con la intención de evitar las disposiciones legales existentes sobre las drogas de abuso ${ }^{2,4-6}$. Este término, aunque desafortunado, es ampliamente aceptado. No obstante, su nombre en español debería ser "drogas del diseñador", si se acepta la traducción del inglés (designer drugs) ${ }^{7}$.

Su expansión comenzó en la década de los sesenta, con la aparición del movimiento hippy en California, llegando a España en los ochenta y pasando de ser prácticamente desconocidos a ponerse de moda en una parte de los jóvenes, preferentemente los fines de semana ${ }^{1,5,6,8,9}$. Posiblemente las ofensivas terapéuticas y preventivas desarrolladas en los últimos años para reducir el consumo de las drogas clásicas y sobre todo el miedo a la transmisión del VIH, son algunas de las causas del inicio del consumo de estas sustancias $^{10}$. En los últimos diez años, desde el denominado "verano del amor" inglés de 1988, el consumo de drogas de diseño, y muy especialmente el de éxtasis, se

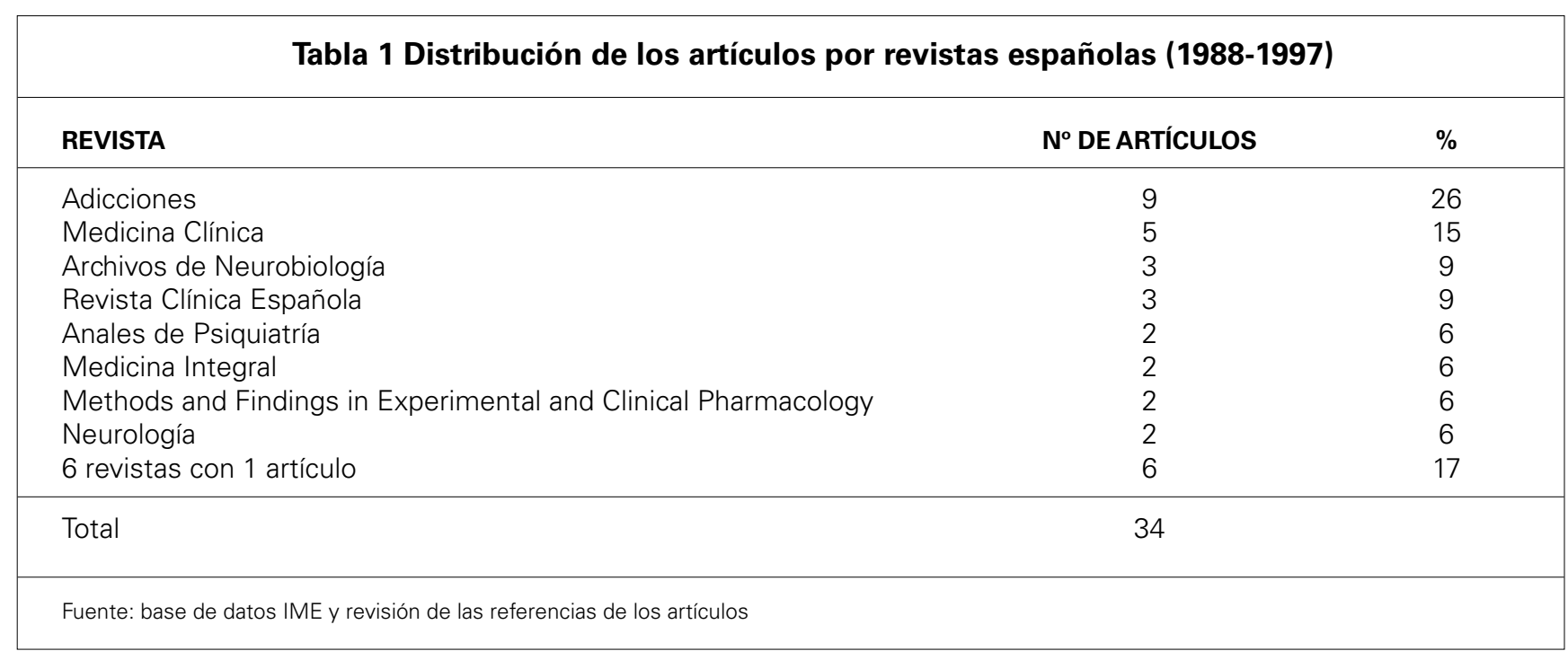

\begin{tabular}{|lc|}
\hline \multicolumn{2}{|c|}{ Tabla 2 Distribución por temas de los artículos publicados en revistas españolas (1988-1997) } \\
\hline TEMA TRATADO* & No DE ARTícULOS \\
\hline Toxicidad & 15 \\
Concepto y clasificación & 10 \\
Actividad farmacológica & 7 \\
Parkinson inducido por MPTP & 6 \\
Mecanismo de acción & 5 \\
Fuente: base de datos IME y revisión de las referencias de los artículos & \\
\hline & * Otros temas tratados son: perfil del consumidor, consumo, epidemiología, psicopatología, aspectos médico-legales, aspectos históricos, antropología, pre- \\
\hline
\end{tabular}


Tabla 3 Trabajos españoles citados en los artículos publicados en revistas nacionales (1988-1997)

\begin{tabular}{|c|c|c|c|}
\hline PUBLICACIÓN & AUTORES & REFERENCIA & $\mathbf{N}^{0}$ DE CITAS \\
\hline Síntesis clandestina de drogas. Presente, pasado y futuro & Camí J & Med Clin (Barc) 1990; 95 & 11 \\
\hline Drogas de diseño ¿un nuevo reto? & Camí J & $\begin{array}{l}\text { Avances en Terapéutica. } 16^{\text {a }} \text { ed., } \\
\text { Salvat, } 1992\end{array}$ & 7 \\
\hline MDMA (éxtasis): droga de actualidad en España & Prat y cols. & Rev Clin Esp 1991; & 5 \\
\hline Plan Nacional sobre Drogas & Plan Nacional sobre Drogas & Memoria (diversos años) & 5 \\
\hline MDMA (éxtasis): revisión y puesta al dia & Blasco Mascaró y cols. & Rev Esp Drogodepend 1991; & 4 \\
\hline Extasis, la droga de la ruta del bakalao & Camí J & Med Clin (Barc) 1996; 106: 711 & 3 \\
\hline Drogas de diseño & García S y cols. & Med Integral 1993; 21: & 3 \\
\hline Extasis (sustancias entactógenas) & Bobes J & $\begin{array}{l}\text { Barcelona: Ediciones en } \\
\text { Neurociencias; } 1995\end{array}$ & 2 \\
\hline Methyl-phenyl-tetrahydropyridine neurotoxicity... & Camí J & Boca Ratón: CRC Press, Inc. & 2 \\
\hline Farmacología y toxicología del MDMA (éxtasis) & Camí J & $\begin{array}{l}\text { Barcelona: Ediciones en } \\
\text { Neurociencias; } 1995\end{array}$ & 2 \\
\hline $\begin{array}{l}\text { Complicaciones neurológicas de las toxicomanías. } \\
\text { Aspectos generales. Complicaciones por cannabis, drogas } \\
\text { de diseño y sustancias volátiles }\end{array}$ & Farré Albadalejo M & Arch Neurobiol 1989; 389-404 & 2 \\
\hline $\begin{array}{l}\text { Drogas de diseño, consumo y consumidores: análisis de la } \\
\text { demanda al servicio telefónico de drogodependencias de la } \\
\text { Generalitat Valenciana }\end{array}$ & Llopis Llácer JJ y cols. & Adicciones 1994; 6: 389-404 & 2 \\
\hline Anfetaminas de anillo sustituido & Elverdín C, Pérez MP & $\begin{array}{l}\text { Panorama Actual del } \\
\text { Medicamento 1992; 154: 278-280 }\end{array}$ & 2 \\
\hline 9 publicaciones con 1 cita & & & 9 \\
\hline TOTAL & & & 64 \\
\hline
\end{tabular}

Tabla 4. Distribución por tipo de artículo publicado

TIPO DE ARTícULO

Artículos originales

Artículos de revisión

Cartas

Editoriales

Ensayos clínicos

Otros*

Total

Fuente: MEDLINE (1988-1997)

No DE ARTí́CULOS
1.763
278
101
8
14
17
2.181

PORCENTAJE

$80,8 \%$

$12,8 \%$

4,6

0,4

0,6

0,8

* Incluye comentarios, noticias, entrevistas, biografías e informes de congresos

ha expandido por bastantes países europeos, junto con la subcultura tecno o house, conocida popularmente en España como "música máquina" o simplemente bakalao. En España el fenómeno se inició y se consolidó a lo largo de la costa mediterrénea, en la que popularmente se conoce como "ruta del bakalao". Este período inicial puede situarse entre 1986 y 1989. Según el informe del Plan Nacional sobre Drogas de $1996^{11}$ el consumo de éxtasis y de otros derivados se ha extendido sobre todo a partir de 1992.

Se consumen preferentemente por vía oral, en forma de comprimidos que suelen llevar grabados nombres o dibujos. Generan efectos psicoestimulan- tes (euforia, empatía, alegría, elevada sociabilidad) que recuerdan los del LSD, pero sin que se alcancen las típicas alteraciones de la percepción de este alucinógeno ${ }^{9}$. El motivo fundamental de su rápida expansión y del incremento de su popularidad y consumo es la falsa idea, tanto social como personal del consumidor, de que tienen una toxicidad mínima o nula, la convicción de obtener mejores relaciones interpersonales y de divertimento, así como el supuesto efecto afrodisí$\mathrm{aco}^{12}$.

Aparte de la toxicidad propia del compuesto alucinógeno, las drogas sintetizadas en laboratorios clandestinos tienen una toxicidad potencial adicional, ya 
Tabla 5. Distribución por revistas de los artículos publicados

REVISTA

Nº DE ARTÍ́CULOS

ÁREATEMÁTICA

Brain Research

European Journal of Pharmacology

157

Journal of Pharmacology and Experimental Therapeutics

102

Journal of Neurochemistry

Neuroscience Letters

Pharmacology, Biochemistry and Behavior

Neuroscience

Annals of the New York Academy of Sciences

Life Sciences

Advances in Neurology

Experimental Neurology

British Medical Journal

Biochemical Pharmacology

Movement Disorders

Neurology

Psychopharmacology

Annals of Neurology

Neuropharmacology

NIDA Research Monograph

Neuroreport

Chemical Research in Toxicology

Biulleten Eksperimentalnoi Biologii i Meditsiny

Journal of Neural Transmission. Supplement

Synapse

Journal of Medical Chemistry

Pharmacology and Toxicology

Neurochemistry International

Journal of Neural Transmission. Parkinsons Disease

Journal of Neuroscience

Neurochemical Research

Brain Research. Molecular Brain Research

Neurotoxicology

Forensic Science International

Journal of Neural Transmission

Journal of Neuroscience Research

Progress in Brain Research

Neuropsychopharmacology

Lancet

Brain Research Bulletin

Journal of Analytical Toxicology

Journal of the Neurological Sciences

Anaesthesia

Toxicology Letters

Toxicology

Clinical Neuropharmacology

Neurodegeneration

Experimental Brain Research

Science

Neurotoxicology and Teratology

Toxicology and Applied Pharmacology

364 revistas con menos de 10 artículos

TOTAL

80

79

77

67

41

37

37

33

31

30

28

28

28

28

27

25

24

23

23

21

21

21

18

18

16

16

15

15

15

15

15

14

14

13

13

13

12

12

11

11

11

11

10

10

10

10

10

10

786

N

F

F

N

N

F

N

G

G

N

N

G

F

N

N

N

N

Ny F

D

N

$\mathrm{T}$

L

N

N

B

Fy $\mathrm{T}$

N

N

N

N

N

N y $T$

MF

N

N

N

$\mathrm{N}$ y $\mathrm{F}$

G

N

T

N

A

T

T

Ny $\mathrm{F}$

N

N

G

N

Ty F

2.181

Fuente: MEDLINE (1988-1997)

$N^{\circ}$ total de revistas: 414

(A: anestesiología; B: bioquímica; D: drogodependencias; F: farmacología; G: medicina general e interna; L: Biología; MF: medicina forense; N: neurología y psiquiatría; T: toxicología) 
Tabla 6. País de procedencia de los trabajos recogidos en MEDLINE (1988-1997)

\begin{tabular}{|c|c|c|}
\hline PAÍSES & Nº DE ARTÍCULOS & PORCENTAJE \\
\hline Estados Unidos & 939 & 43,4 \\
\hline Holanda & 409 & 18,75 \\
\hline Gran Bretaña & 388 & 17,8 \\
\hline Alemania & 95 & 4,35 \\
\hline Austria & 61 & 2,8 \\
\hline Irlanda & 51 & 2,4 \\
\hline Rusia & 44 & 2 \\
\hline Japón & 35 & 1,6 \\
\hline Dinamarca & 29 & 1,3 \\
\hline España & 20 & 0,9 \\
\hline Suiza & 18 & 0,8 \\
\hline China & 15 & 0,7 \\
\hline Australia & 14 & 0,6 \\
\hline Canadá & 9 & 0,4 \\
\hline Francia & 9 & 0,4 \\
\hline Italia & 8 & 0,2 \\
\hline Polonia & 5 & 0,2 \\
\hline Nueva Zelanda & 4 & 0,2 \\
\hline Singapur & 4 & 0,1 \\
\hline Bélgica & 3 & 0,1 \\
\hline Israel & 3 & 0,1 \\
\hline Taiwan & 3 & 0,1 \\
\hline Checoslovaquia & 2 & 0,1 \\
\hline Noruega & 2 & 0,1 \\
\hline Sudáfrica & 2 & 0,1 \\
\hline Suecia & 2 & 0,1 \\
\hline 7 países con 1 artículo & 7 & 0,4 \\
\hline TOTAL & 2.181 & \\
\hline
\end{tabular}

Tabla 7 Principales aspectos tratados en los artículos recogidos en la base de datos MEDLINE (1988-1997)

\section{ASPECTOTRATADO}

Nº DE ARTíCULOS

Toxicidad de la 1-metil-4-fenil-1,2,3,

6-tetrahidropiridina 229

Análogos y derivados de la MDMA 226

Farmacología de la 1-metil-4-fenil-1,2,3,

6-tetrahidropiridina $\quad 210$

Metabolismo de la dopamina 204

Metabolismo del corpus striatum 122

Enfermedad de Parkinson inducida químicamente 121

Metabolismo cerebral 109

Análogos y derivados de la 1-metil-4-fenil-1,2,

3,6-tetrahidropiridina

Efectos de las drogas sobre el cerebro

Fisiología de la dopamina $\quad 88$

Fisiopatología de la enfermedad de Parkinson $\quad 86$

Efectos de las drogas sobre el corpus striatum $\quad 79$

Metabolismo de la serotonina $\quad 79$

Farmacología de los agentes dopaminérgicos $\quad 76$

Farmacología de la 3,4-metilenedioxianfetamina 73

Efectos de las drogas sobre las neuronas $\quad 73$

Farmacología de las anfetaminas $\quad 65$

Toxicidad de las piridinas $\quad 65$

Metabolismo de la enfermedad de Parkinson $\quad 63$

Efectos de las drogas sobre la sustancia negra $\quad 62$

Metabolismo de la monoamino oxidasa $\quad 59$

Farmacología de la N-Metil-3,4-metilenedioxianfetamina

Efectos de las drogas sobre la actividad motora

Drogas de diseño

Farmacología de las pridinas

Farmacoterapia de la enfermedad de Parkinson

1-metil-4-fenil-1,2,3,6-tetrahidropiridina

Fuente: descriptores (términos MeSH) de MEDLINE

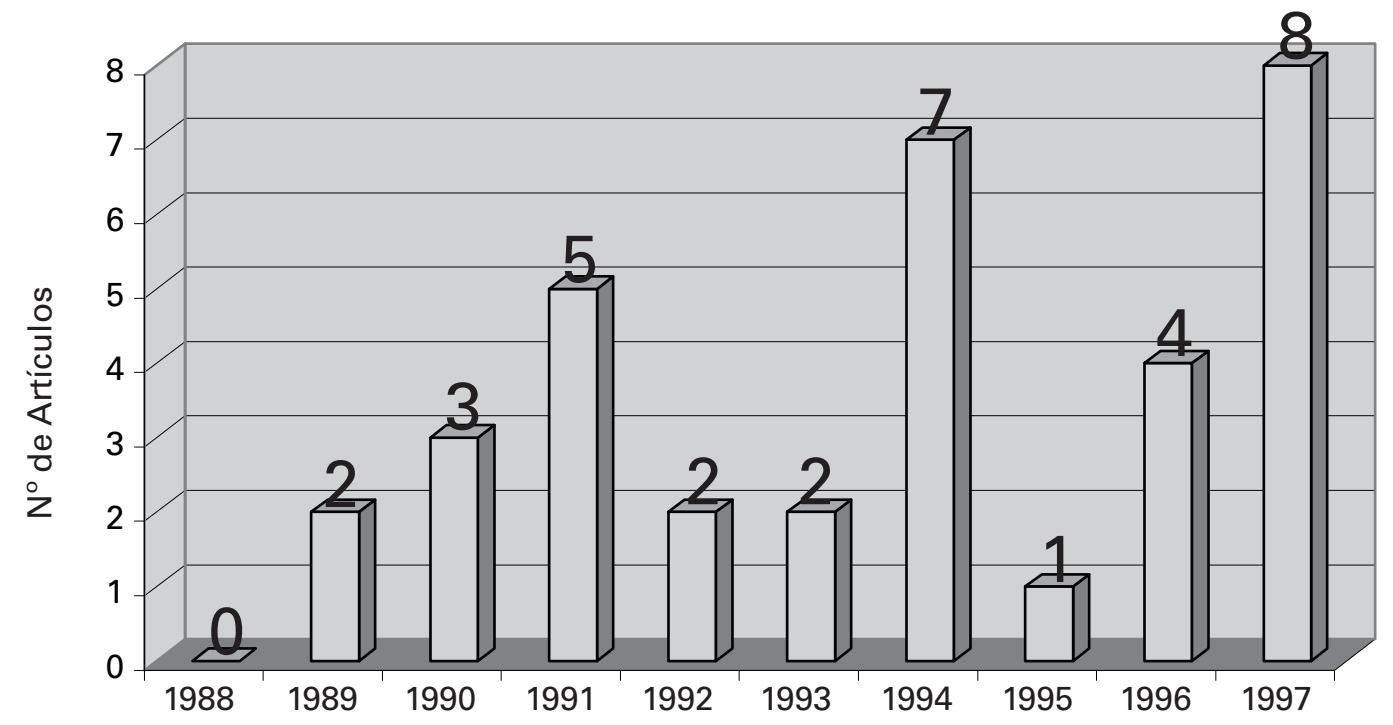

Figura 1. Distribución por años de las publicaciones españolas (1988-1997)

Fuente: base de datos IME 
Tabla 8 Instituciones con 10 o más trabajos de las publicaciones recogidas en MEDLINE (1988-1997)

\section{INSTITUCIÓN}

NNo DE ARTíCULOS

University of California, Los Angeles, CA, USA

55

National Institute of Mental Health, Bethesda, MD, USA

Hahnemann University School of Medicine, Philadelphia, PA, USA 35

University of Utah, Salt Lake City, UT, USA $\quad 26$

King's College, London, UK

University of Medicine and Dentistry of New Jersey, Robert Wood Johnson

Medical School, Piscataway, NJ, USA

24

University of Rochester School of Medicine, NY, USA

Yale University School of Medicine, New Haven, CT, USA

Johns Hopkins University School of Medicine, Baltimore, MD, USA 22

Nagoya University School of Medicine, Japón

Virginia Polytechnic Institute and State University, Blacksburg, USA

Hopital de I-Enfant-Jesus, Quebec City (Quebec), Canadá 19

Northeastern Ohio Universities College of Medicine, Rootstown, USA 19

School of Pharmacy and Pharmacal Sciences, Purdue University, West Lafayette, IN, USA 18

National Center for Toxicological Research, Jefferson, Arkansas, USA 17

NIDDK, National Institute of Health, Bethesda, MD, USA 16

University of Wurzburg, Alemania $\quad 16$

NIDA, Addiction Research Center, Baltimore, MD, USA 15

Karolinska Institute, Stockholm, Suecia 14

Case Western Reserve University School of Medicine, Cleveland, OH, USA 13

Massachusetts General Hospital, Boston, USA $\quad 13$

Mount Sinai School of Medicine, New York, NY, USA 13

Ohio State University, College of Medicine, Columbus, OH, USA 13

University of Texas Southwestern Medical Center, Dallas, USA $r$

University of Toronto, Ontario., Canadá 12

Eli Lilly and Company, Lilly Corporate Center, Indianapolis, IN, USA 11

Juntendo University School of Medicine, Tokyo, Japón 11

Lilly Research Laboratories, Eli Lilly and Company, Indianapolis, IN, USA 11

Merrey Dow Research Institute, Cincinnati, OH, US 11

Okayama University Medical School, Japón 11

Trinity College, Dublin, Irlanda $\quad 11$

U.S. Environmental Protection Agency, Research Triangle Park, USA 11

Hopital de la Salpetriere, París, Francia $r$

Laval University, Quebec, Canadá $\quad 10$

New York University Medical Center, NY, USA $r$

Royal Free Hospital School of Medicine, Londres, UK 10

University of Miami School of Medicine, Florida, USA 10

University of Michigan Medical School, Ann Arbor, USA 10

que pueden presentar restos de los precursores y reactivos no controlados utilizados en su síntesis (metil-dioxi-benzilacetona), reacciones químicas colaterales, productos intermedios y sustancias añadidas para adulterar la droga que son, en muchos casos, más tóxicos que la propia droga ${ }^{13,14}$.

El objetivo de este trabajo es analizar la producción científica nacional e internacional sobre drogas de diseño, a partir del análisis bibliométrico de las publicaciones. Se pretende identificar las principales fuentes de información, así como las características de las publicaciones y las de los investigadores y grupos que las generan. Por otra parte, el análisis bibliométrico permite detectar las lagunas en la investigación, información que puede ser aprovechada por los responsables de la política científica para potenciar las áreas con escasa actividad investigadora y optimizar la asignación de recursos.

\section{MATERIAL Y METODO}

Para conocer la producción científica nacional e internacional sobre drogas de diseño, se han interrogado las bases de datos Indice Médico Español (IME) y MEDLINE durante el período 1988-1997. La base de datos IME incluye la mayor parte de las revistas médicas españolas de carácter científico (143 revistas en 1997). La base de datos MEDLINE es la más ampliamente utilizada en el ámbito internacional, ya que analiza más de 3.200 revistas de carácter biomédico. La 

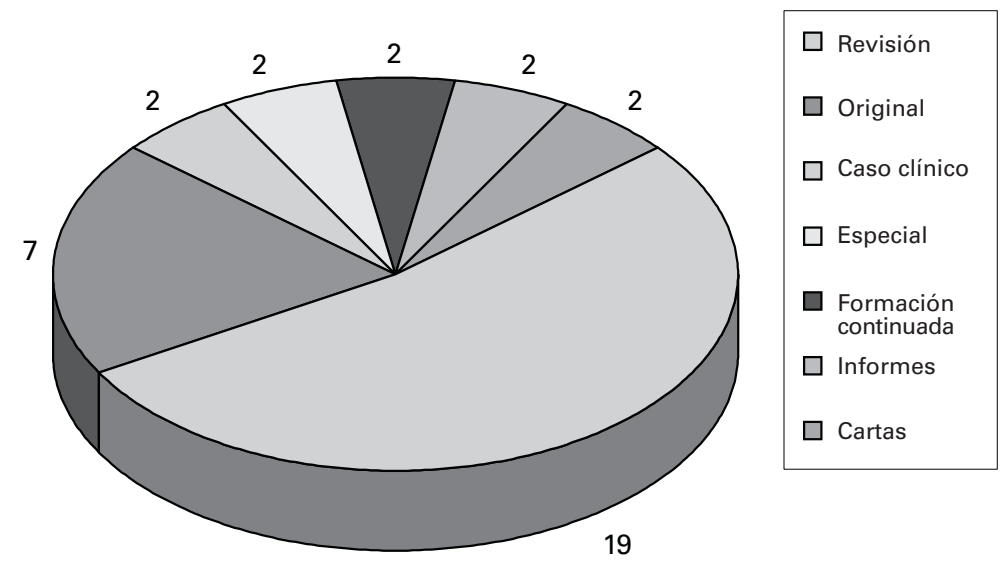

Figura 2. Distribución por tipo de trabajos de las publicaciones españolas (1988-1997)

Fuente: base de datos IME

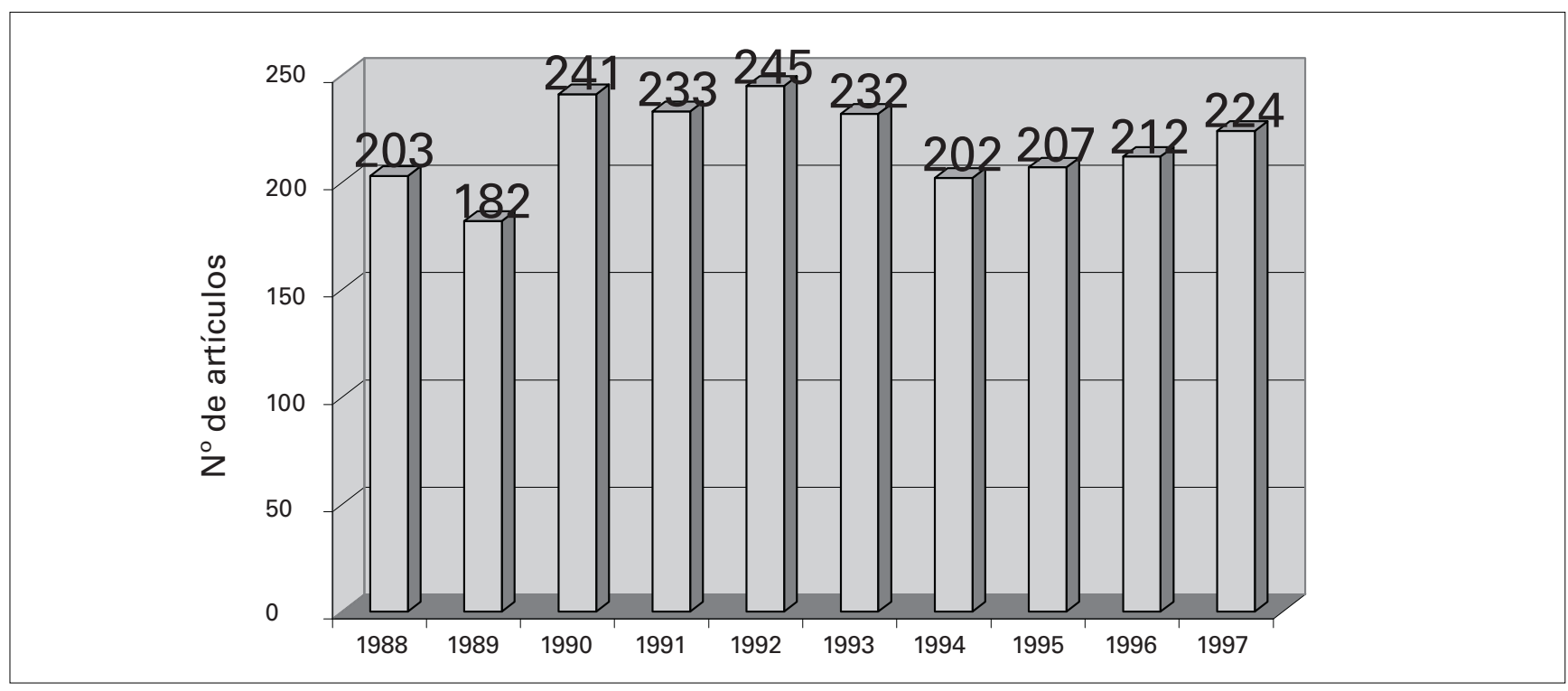

Figura 3. Distribución por años de las publicaciones recogidas en MEDLINE (1988-1997)

Fuente: base de datos IME

búsqueda en MEDLINE se realizó utilizando el vocabulario controlado del Medical Subject Headings (MeSH), lo que garantizaría la pertinencia de los resultados obtenidos. De acuerdo con las instrucciones del $\mathrm{MeSH}$, los términos que se emplearon en el perfil de búsqueda fueron: 3,4-metilenedioxianfetamina; MDMA; MPTP y designer drugs.

Dado el carácter interdisciplinar de las drogodependencias, se interrogó también la base de datos del Indice Español de Ciencias Sociales (ISOC), en la que se recogen la mayor parte de las revistas españolas de psicología, psiquiatría y otras áreas temáticas que podrían ser de interés, como criminología y derecho. Por último, se hizo una búsqueda en la base de datos teseo del Ministerio de Educación y Ciencia para obte- ner información sobre la tesis doctorales presentadas en España.

El perfil de búsqueda utilizado en las bases de datos españolas (IME e ISOC) estaba constituido por términos genéricos (como drogas de diseño y drogas de síntesis), términos específicos (como metanafetamina, 1-metil-4-fenil-1,2,3,6-tetrahidropiridina, etc.), abreviaturas comunes (tales como MDMA, MDEA, etc.) y términos del argot (como éxtasis, Eva, Adán, etc.).

Para lograr una población de artículos españoles lo más exhaustiva posible se revisaron las referencias bibliográficas de los 31 artículos recuperados en la primera fase en la base de datos IME. Gracias a esta labor se identificaron otros tres artículos publicados en las revistas Neurología, Panorama Actual del Medi- 


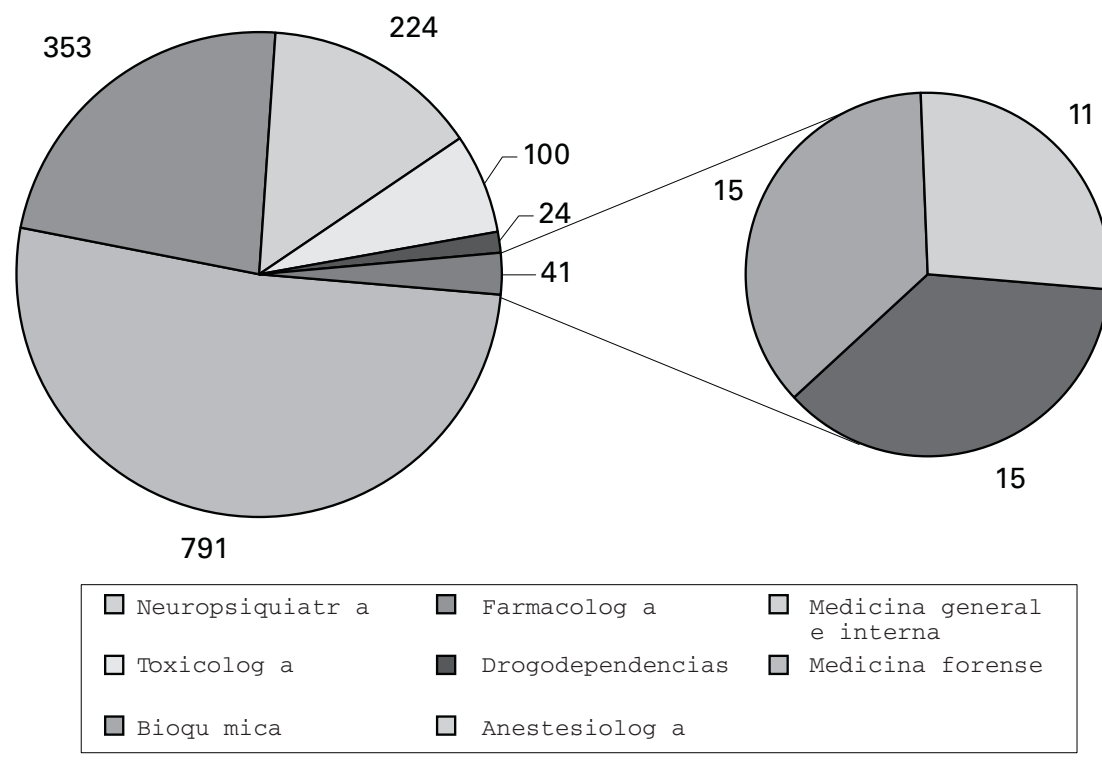

Figura 4. Distribución por áreas temáticas de las publicaciones recogidas en MEDLINE (1988-1997)

Fuente: base de datos IME

camento y Revista de Sanidad e Higiene Pública. Estos artículos no fueron incluidos posiblemente por su carácter no científico, pues se trataba de cartas al director e informes. En la base de datos ISOC se obtuvieron cuatro artículos nuevos, publicados en las revistas Cuadernos de Política Criminal, Ciencia Policial, Información Psicológica y Psicopatología. El análisis de las referencias también ha permitido identificar las publicaciones españolas más citadas sobre esta temática.

Los artículos obtenidos se clasificaron según el año de publicación, tipo documental, revista de publicación, temática de la revista, instituciones y países de procedencia, idiomas y temas tratados (de acuerdo con los descriptores asignados por los analistas de la base de datos). Se determinó también el índice de colaboración (media del número de autores por trabajo). El impacto de las revistas se obtuvo consultando el Journal Citation Reports de $1996^{15}$.

\section{RESULTADOS}

\section{Publicaciones en revistas españolas}

En las revistas españolas se publicaron 34 artículos sobre el tema (36 si se consideran dos cartas al director), siendo en 1994 y 1997 cuando se publicaron más artículos (7 y 8, respectivamente) (figura 1). Los tipos de trabajo predominantes fueron los artículos de revisión y los artículos originales, con 19 y 7 trabajos, respectivamente (figura 2). En las tablas 1 y 2 se distribuyen los artículos por revistas y temas tratados, respectivamente. Las revistas que más artículos han publicado son Adicciones y Medicina Clínica. Los principales aspectos tratados en estos artículos se refieren a la toxicidad, concepto y clasificación y actividad farmacológica de las drogas de diseño. Las publicaciones más citadas en estos artículos se muestran en la tabla 3. Destaca el artículo "Síntesis clandestina de drogas: pasado, presente y futuro", publicado en Medicina Clínica en 1990, que ha recibido 11 citas, y la monografía Drogas de diseño ¿un nuevo reto?, editada por Salvat en 1992, ambas publicadas por Camí.

\section{Publicaciones internacionales}

En la figura 3, que muestra la distribución por años de los 2.181 artículos recuperados, no se aprecia ninguna tendencia al crecimiento sino más bien una constante anual en torno a los 200 artículos. En la tabla 4, en la que se distribuyen los artículos según su tipo documental, puede apreciarse que el $80 \%$ de los artículos son originales y el 13\% artículos de revisión. En la tabla 5 se presentan las revistas más productivas (con 10 o más trabajos), así como sus áreas temáticas. Las revistas que más artículos aportan son Brain Research y European Journal of Pharmacology. Las áreas temáticas de las revistas se pueden apreciar en la figura 4, en la que se observa que las áreas más productivas son la neuropsiquiatría, la farmacología y la medicina general e interna, con el 36\%, 16\% y $10 \%$, respectivamente. La tabla 6 presenta la distribución por países de los artículos. La mayor parte proceden de los Estados Unidos, con el 43\%, seguido de Holanda y Gran Bretaña, con porcentajes cercanos al $20 \%$. Respecto a los aspectos o temas tratados en los artículos (tabla 7), destacan los relacionados con la toxicidad y farmacología de la1-metil-4-fenil-1,2,3,6- 
tetrahidropiridina, los análogos y derivados de la MDMA, así como los efectos de estas drogas sobre el metabolismo cerebral y la enfermedad de Parkinson inducida químicamente. Los centros que más trabajos aportan proceden, principalmente, de los Estados Unidos, Gran Bretaña y Japón (tabla 8). Las instituciónes que más trabajos aportan son la University of California y el National Institute of Mental Health.

\section{Tesis doctorales}

En cuanto a las tesis doctorales, en el último decenio se presentaron en España ocho tesis relacionadas con esta temática. En seis de ellas se investigaron diversos aspectos de la Enfermedad de Parkinson inducido por MPTP y, en las otras dos, la neurotoxicidad de la MDMA y las técnicas analíticas aplicadas a las drogas de diseño, respectivamente. Cuatro tesis fueron presentadas en la Universidad de Navarra y tres en la de Barcelona.

\section{DISCUSION}

La revisión documental de los artículos españoles analizados indica que los problemas que plantea el consumo de drogas de diseño son:

- el importante consumo entre la población escolar y juvenil.

- el discurso justificativo de sus consumidores, que favorece su expansión ${ }^{17}$ : el consumo de estas drogas disminuye el de alcohol; se trata de drogas modernas y democráticas que favorecen más la sensualidad que la sexualidad (con lo que se previene el SIDA); intensifican la comunicación y solidaridad grupal.

- las dificultades para su intervención y detección y, por consiguiente, menor eficacia en su control ${ }^{18-20}$.

- su toxicidad6: trastornos neuropsicológicos: depresión, ansiedad, psicosis, hepatitis, golpe de calor. Por otra parte, se desconocen los efectos y la toxicidad de las nuevas drogas que se van diseñando, así como los efectos de éstas a largo plazo.

- la toxicidad potencial adicional como consecuencia de la adulteración, que ha aumentado desde su prohibición, por lo que algunos ven en su legalización la solución a muchos problemas.

Desde el punto de vista del análisis bibliométrico de los artículos, se confirma que existen pocos trabajos españoles publicados sobre drogas de diseño en comparación con la producción global en drogodependencias21, y los que hay suelen ser revisiones, estados de la cuestión o puesta al día sobre el concepto y clasificación de estas drogas.
La ilegalidad de estas sustancias dificulta en ocasiones la realización de estudios experimentales y epidemiológicos, pudiéndose ser este el motivo del escaso número de ensayos clínicos realizados (0,6\%). Por el contrario, en el ámbito internacional predominan los artículos originales, por lo que puede deducirse que la investigación sobre el tema está más consolidada en el extranjero, mientras que en España se limita a revisar y sintetizar la información que llega de fuera. En cualquier caso, se ha advertido, tras la revisión de todos los artículos, que gran parte de los que se publican bajo el epígrafe de originales, en realidad su contenido no se corresponde con el de artículos científicos estructurados de acuerdo con la metodología de la investigación y más bien podría enmarcarse dentro de los artículos de revisión, actualización, o incluso como artículos de opinión. La producción anual de trabajos parece aumentar en el caso español, manteniéndose constante la internacional.

En cuanto a las revistas españolas, la más productiva está especializada en drogodependencias (Adicciones) y las demás son de temática general (como Medicina Clínica, Revista Clínica Española y Medicina Integral), neuropsiquiatría (Archivos de Neurobiología y Anales de Psiquiatría) y farmacología (Methods and Findings in Experimental and Clinical Pharmacology). A nivel internacional predominan, por el contrario, las revistas de neuropsiquiatría (Brain Research), lo que indica la importancia de la investigación que realizan neurólogos y psiquiatras en esta área. En un segundo plano, también destacan las revistas que tratan los aspectos farmacológicos y toxicológicos de estas drogas. La principal revista extranjera de esta temática es NIDA Research Monograph. Por último, la presencia de revistas de propósito general se justifica tanto por la propia multidisciplinariedad de esta conducta adictiva en el ámbito médico-sanitario, como por el interés que genera en todos los ámbitos científicos. A partir de este análisis puede concluirse que la temática que más interesa a los investigadores en la materia es la farmacología y la fisiopatología. Por el contrario, no se trata el abordaje terapéutico de los pacientes que presentan problemáticas asociadas al consumo de estas sustancia, así como su prevención.

En líneas generales las líneas de investigación son las mismas tanto en España como en el extranjero, pero mientras que en España los trabajos suelen ser de revisión, en el extranjero predomina más la investigación básica. por otra parte, los países que más publicaciones aportan son Estados Unidos, Holanda y Gran Bretaña, debido tanto a la magnitud de los recursos destinados al área, como a su mayor sensibilización al problema

El índice de colaboración en los artículos españoles se situó en 2,9 autores por trabajo. Este valor es bajo con respecto al encontrado en otras áreas biomédicas, como la toxicología ${ }^{22}$, la dependencia a las dro- 
gas $^{23}$ y la atención primaria ${ }^{24}$. Ello es debido tanto al alto porcentaje de artículos de revisión, que suelen estar firmados por un único autor y, por lo tanto, desvían el índice hacia la unidad, como por la escasa cantidad de originales, donde la colaboración entre varios autores es la norma. Dada la relación existente entre la magnitud de este índice y el grado de apoyo financiero, puede concluirse que existe un deficiente soporte económico para realizar proyectos de investigación sobre esta dependencia ${ }^{25-26}$. Las universidades son los centros que desarrollan una mayor actividad investigadora en el área, lo que se muestra de acuerdo con estudios previos ${ }^{21}$.

En cuanto al idioma de los artículos recogidos en MEDLINE, el 93\% de ellos se ha publicado en inglés, mientras que solamente el $62 \%$ procede de países anglófonos. Este hecho es significativo del creciente papel que desempeña este idioma como vehículo de comunicación científica en el mundo, sobre todo en medicina, donde se está convirtiendo en lingua fran$\mathrm{ca}^{27}$. El fenómeno se produce con mayor intensidad en el área que estamos abordando debido, probablemente, a las preferencias de los autores por publicar en este idioma y lograr así una mayor difusión de sus trabajos.

El análisis temático ha mostrado un predominio de los estudios sobre la 1-metil-4-fenil-1,2,3,6-tetrahidropiridina, sobre todo su toxicidad, farmacología, análogos y derivados. También destacan los trabajos sobre los análogos y derivados de la MDMA y los que estudian el metabolismo de la dopamina, cuerpo estriado y metabolismo cerebral en general. Por último, otros estudios se centran en los efectos de las drogas sobre las neuronas, el cuerpo estriado, la sustancia negra y la actividad motora.

Respecto al análisis de referencias de los artículos publicados en revistas españolas, debe destacarse la repercusión de algunos de los trabajos publicados por Camí, Prat y Blasco Mascaró, que han recibido 11, 5 y 4 citas, respectivamente. Sin entrar a valorar la calidad científica de estos trabajos, que no es el objetivo de este trabajo, otros factores que pueden haber favorecido su citación son el hecho de que se hayan publicado en revistas con amplia difusión en bases de datos nacionales y extranjeras ${ }^{28}$ e incluso con impacto en el Journal Citation Reports del Science Citation Index ${ }^{15}$. Por otra parte, se trata de artículos de revisión o puesta al día, trabajos que generalmente son más citados que otros tipos documentales ${ }^{29}$.

\section{BIBLIOGRAFÍA}

1. Roig-Traver A. Sobre el uso recreativo de la metilendioximetanfetamina: aspectos históricos y efectos adversos. Adicciones 1994; 6: 437-452.
2. Estañ L, Alós M, Villar VM. Concepto y clasificación de drogas de diseño. Farm Clin 1996; 13: 42-54.

3. Jaffe JH. Drogadicción y abuso de drogas. En: Goodman A, Rall TW, Nies AS, Taylor P, eds. Las bases farmacológicas de la terapéutica. $8^{a}$ ed. Nueva York: Pergamon Press, 1991. P. 513-561.

4. Buchanan JF, Brown CR. Designer drugs. A problem in clinical toxicology. Med Toxicology 1988; 3: 1-17.

5. Camí J. Farmacología y toxicidad de la MDMA (éxtasis). Barcelona: Ediciones en Neurociencias, 1995.

6. Camí J, Farré M. Éxtasis: la droga de la ruta del bakalao. Med Clin (Barc) 1996; 106: 711-716.

7. Martínez M. Drogas de diseño. Med Mil 1993; 49: 42-49.

8. Prat A, Montero M, Reig R, Sanz P. MDMA ("éxtasis"): droga de actualidad en España. Rev Clin Esp 1991; 188: 106-108.

9. Nogué S, Sanz-Gallén P. Drogas de diseño. Med Integral 1997; 29: 138-144.

10. Llopis JJ, París B, Bojó P, Camarena F, Malea A, González $S$, et al. Drogas de diseño, consumo y consumidores: análisis de la demanda al servicio telefónico de drogodependencias de la Generalitat Valenciana. Adicciones 1994; 6: 389-404.

11. Delegación del Gobierno para el Plan Nacional sobre Drogas. Memoria 1996. Madrid: Ministerio de Justicia e Interior; 1997.

12. Cabrera R, del Río PA. Drogas anfetamínicas de diseño. Revisión toxicológica. Rev Toxicol 1994; 11: 49-57.

13. Fernández PL. MDMA (éxtasis). Una droga de diseño de alta toxicidad potencial. Adicciones 1994; 6: 405-422.

14. García S, Geijo S, Álvarez FJ. Drogas de diseño. Med Integral 1993; 21: 300-309.

15. Journal Citation Reports 1996. Filadelfia: Institute for Scientific Information; 1998.

16. Sueur C. Consumo de éxtasis en Francia: prevención de los riesgos en las "raves" y tratamiento de las complicaciones psicopatológicas. Adicciones 1997; 9 (3): 445-456.

17. Calafat A, Sureda MP, Palmer A. Características del consumo de éxtasis en una muestra de universitarios y usuarios de discotecas. Adicciones 1997; 9: 529-555.

18. Camí J. Síntesis clandestina de drogas: pasado, presente y futuro. Med Clin (Barc) 1990; 95: 344-347.

19. Camí J. Síntesis clandestina de drogas. Política Científica 1989; 19: 18-21.

20. Jerrard DA. Designer drugs: a current perspective. J Emerg Med 1990; 8: 733-741.

21. Valderrama JC. Producción científica en toxicomanías. Ponencia presentada a la $10^{a}$ Reunión CITRÁN "Adicción a opiáceos". Sitges; 1998.

22. Guardiola E, Camí J (a). La investigación toxicológica en España vista a través de las bases de datos 1985-1986. Med Clin (Barc) 1988; 91: 11-15.

23. Guardiola E, Camí J (b). Difusión internacional de las investigaciones españolas en dependencia a las drogas. Med Clin (Barc) 1988; 91: 375-378.

24. Aleixandre R, Porcel A, Agulló A, Marset S, Abad F. Diez años de la revista Atención Primaria (1984-1993). Análi- 
sis bibliométrico y temático. Aten Primaria 1996; 17 : 225-230.

25. Beaver DB, Rosen R. Studies in scientific collaboration. Part I. The professional origins of scientific co-autorship. Scientometrics 1978; 1: 65-84.

26. Pao ML. Global and local collaborators: a study of scientific collaboration. Inf Proc Manag 1992; 28: 99-109.
27. Aleixandre R, Porcel A, Agullo A, Marset S. Vicios del lenguaje médico. I. Extranjerismos y acrónimos. Aten Primaria 1995; 15: 113-118.

28. López-Piñero JM, Terrada ML. La información científica en medicina y sus fuentes. Valencia: Instituto de Estudios Documentales e Históricos sobre la Ciencia; 1993.

29. Guardiola E. El artículo de revisión: hacia un mayor rigor científico. Rev Salud Publica 1991; 2: 197-218. 
